Ann. Biol. anim. Bioch. Biophys., I972, $12(4), 559-567$.

\title{
RÔLE DES SURRÉNALES MATERNELLES DANS LA PRODUCTION D'ESTROGENES PAR LA TRUIE GRAVIDE
}

\author{
J. FÈVRE, P.-C. LÉGLISE et Odette REYNAUD \\ avec la collaboration technique de J. SuREL et de F. JAOuen \\ Station centrale Physiologie animale, \\ Centre national de Recherches zootechniques, I. N. R. A., \\ 78350 Jouy en Josas
}

\section{RÉSUMÉ}

Chez la Truie, les quantités d'œstrone excrétées dans l'urine, augmentent considérablement à la fin du premier mois de gestation et à partir du $75^{\mathrm{e}}$ jour. Pour connaître l'importance des stéroïdes surrénaliens maternels dans la synthèse des oestrogènes par les unités foeto-placentaires, nous avons surrénalectomisé quatre Truies à divers moments de leur gestation (tabl. I).

La technique chirurgicale de surrénalectomie est décrite. Elle est toujours pratiquée en deux temps : surrénalectomie droite d'abord, suivie au moins zo jours plus tard par la surrénalectomie gauche. La survie des animaux est obtenue grâce à l'administration quotidienne d'acétate de désoxycorticostérone, de chlorure de sodium et de chlorure de potassium, l'absence de thérapeutique substitutive entraînant la mort des animaux.

Après surrénalectomie totale, l'excrétion urinaire d'œstrone présente, comme chez les animaux gravides intacts, un maximum autour du $28 \mathrm{e}$ jour de gestation (fig. 2 et 3 ). En fin de gestation, nous trouvons les valeurs maximales de l'élimination urinaire d'oestrone, ces valeurs étant les mêmes qu'il y ait eu surrénalectomie ou non (fig. 4,5 et 6 ).

Chez la Femme, 1'ensemble des travaux montre que les surrénales maternelles sécrètent au moins la moitié des précurseurs nécessaires à la biosynthèse des oestrogènes pendant la grossesse. Nos résultats montrent, au contraire, que chez la Truie cette synthèse par les unités foeto-placentaires peut s'effectuer normalement en l'absence de précurseurs stéroïdiens maternels.

\section{INTRODUCTION}

Chez la Truie, comme chez d'autres espèces, la synthèse des œestrogènes, au cours de la gestation, est le fait essentiellement des unités fœto-placentaires. Nous avons en effet montré qu'après ovariectomie la production d'œstrogènes reste normale, même à la fin du premier mois de gestation (FìVRE, LÉGLISE, RombaUTS, I968). II a été également constaté que la conversion en œstrone de quelques stéroïdes 
C-I9 par la Truie gravide est plus importante lorsque ces stéroïdes sont administrés dans le compartiment fœetal (FÈVRE, 1970). De même, nous avons pu voir depuis que la $\mathrm{x}_{7} \alpha$-hydroxyprogestérone- $7 \quad \alpha-{ }^{3} \mathrm{H}$ injectée à la mère n'est pas convertie en cestrone, alors que son taux de conversion est de 4,I3 p. I0o en cas d'administration intra-amniotique (résultats non publiés). L'hypophysectomie de la Truie entraîne une certaine régression pondérale des surrénales et, cependant, l'excrétion urinaire d'œstrone reste très élevée pendant la gestation (FÈVRE, LÉGLISE, ROMBAUTS, I968). Il semblerait ainsi que l'ensemble foeto-placentaire porcin n'utiliserait pas (ou peu) des précurseurs stéroïdiens d'origine maternelle pour effectuer 1a synthèse des œstrogènes.

Pour connaître la contribution exacte des stéroïdes surrénaliens maternels dans la biosynthèse des œestrogènes par l'unité fœeto-placentaire porcine, nous avons suivi l'excrétion urinaire d'œestrone chez des Truies gravides surrénalectomisées en début ou en fin de leur gestation.

\section{MATÉRIEL ETT MÉTHODES}

\section{Matériel animal}

Tous les animaux utilisés étaient des Truies primipares de race Large-White. Ils furent maintenus en cage à métabolisme ce qui a permis de recueillir quantitativement et séparément des fèces, les urines de 24 heures.

\section{Dosage des aestrogènes urinaires}

Les œestrogènes ont été dosés selon une méthode dérivée de celle de BRowN (I955) et décrite dans un article précédent (Fìvre, LEglrse, Rombauts, 1968).

\section{Surrénalectomie de la Truie}

Les surrénalectomies ont été pratiquées sur des Truies gravides. Elles ont toutes été réalisées en deux temps : surrénalectomie droite, puis surrénalectomie gauche. Nous avons cependant tenté de réaliser une surrénalectomie totale en un seul temps par voie médiane sous-xyphoïdienne, mais le jour nettement insuffisant et les difficultés d'approche de la loge rénale rétropéritonéale nous ont fait abandonner cette voie.

Comme KoLB et al. (1962) le remarquent, la surrénalectomie droite présente des problèmes chirurgicaux réels en raison de l'accolement intime de la glande sur le flanc droit de la veine cave inférieure et des variations anatomiques individuelles parmi lesquelles, la plus redoutable (pour la bonne marche de l'intervention), est une disposition hémi-hélicoïdale de la surrénale sur le flanc de la veine cave.

L'anesthésie des animaux mis à jeun la veille de l'intervention est essentiellement gazeuse (oxygène, protoxyde d'azote, cyclopropane), après prémédication d'atropine (20 $\mathrm{mg}$ ), induction barbiturique et intubation. L'animal anesthésié est placé en décubitus latéral gauche sur une table articulée. Les membres postérieurs sont attachés en hyperextension, les antérieurs sont tirés à environ $45^{\circ}$ du plan de la table. Les articulations de la table permettent d'abaisser les régions caudale et céphalique, ce qui impose au tronc de l'animal une courbure qui élève les lombes.

L'incision est semi-circulaire. Elle surcroise les trois dernières côtes et devient, à son extrémité caudale, parallèle à la colonne vertébrale. L'hémostase est réalisée par électrocoagulation. Les plans musculaires dont les fibres croisent l'incision sont sectionnés. Les fibres du carré des lombes sont seulement dilacérées. Les trois dernières côtes sont dénudées, puis sectionnées et les vaisseaux intercostaux coagulés. Le péritoine est décollé jusqu'à la découverte du rein droit et de la veine cave inférieure. Les viscères abdominaux recouverts du péritoine sont alors refoulés 
dans la cavité abdominale à 1'aide de champs humidifiés de sérum physiologique. Des champs de bordure sont ensuite agrafés à la paroi et un cadre d'Auvert est mis en place. Il permet une bonne ouverture du champ opératoire et repousse le foie et l'intestin grêle.

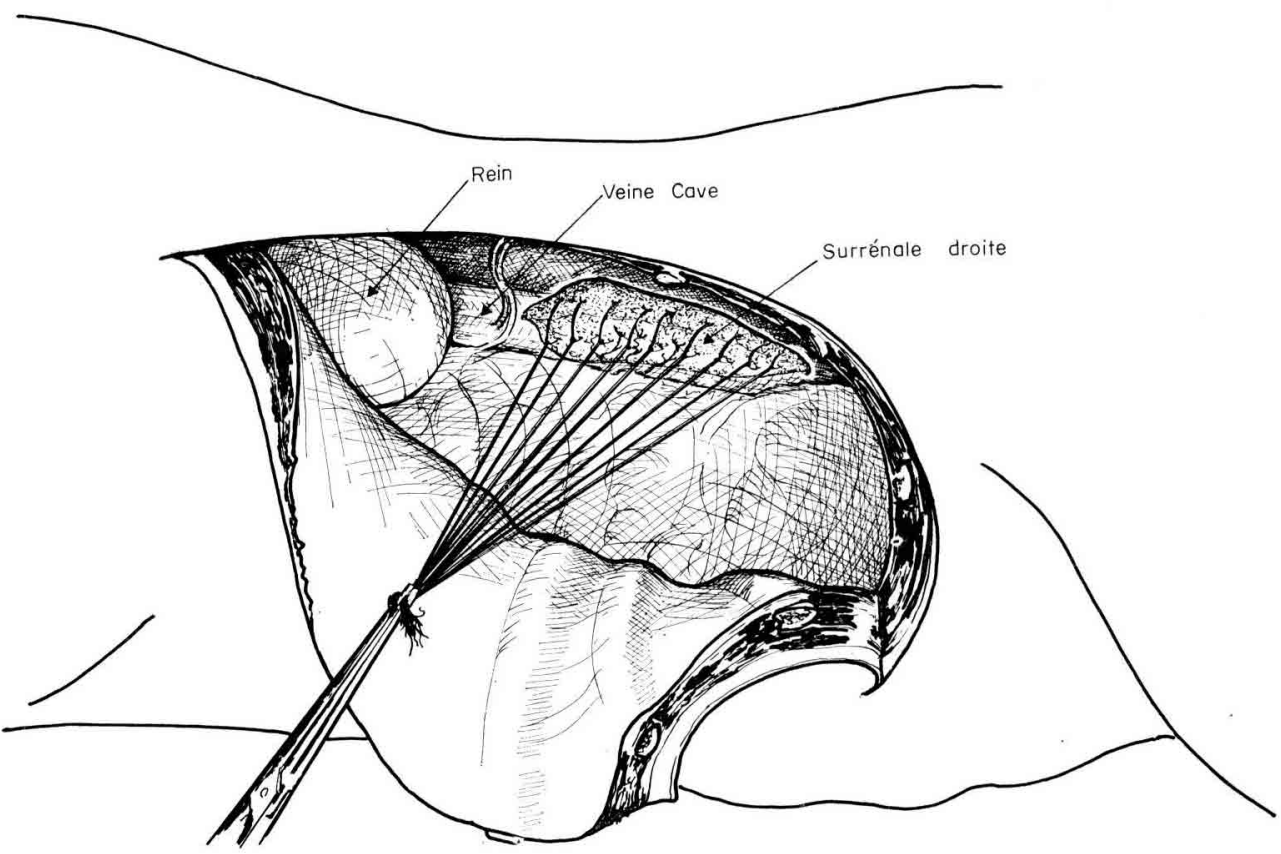

FIG. I. - Surrenalectomie droite de la Truie

Le palper de la surrénale droite, la dissection superficielle des couches adipeuses respectant la gaine conjonctive de la glande permet d'en apprécier ses limites. Il ne nous a cependant pas été possible de trouver un élément de pronostic de la répartition de la vascularisation. Pour cette raison, nous avons choisi pour la dissection de la glande, une technique classique utilisée pour les tumeurs friables. Une dizaine de fils sont passés au travers de la surrénale. Ces fils, pris dans une pince, forment un réseau de traction qui permet l'élévation de la glande sans déchirure, si l'intégrité de la gaine conjonctive a été respectée (fig. I). Utilisant cette traction, on commence le décollement glandulaire par le pôle caudal. Tout vaisseau rencontré est pris dans une pince de Bengolea. Cette dissection peut se faire sur un tiers de la glande. On reprend alors le décollement par le pôle céphalique. A la fin de la dissection, seul le tiers médian de la surrénale reste adhérent à la veine cave. Un clamp de Potts-Satinsky est glissé entre veine et glande et est serré sur le flanc de la veine. La surrénale droite est alors resséquée, entraînant avec elle la portion de la veine cave qui lui est adhérente. Les vaisseaux serrés dans les Bengolea sont électrocoagulés et la brèche ouverte sur le flanc de la veine cave est suturée à la soie vasculaire oooo.

Après vérification de l'hémostase et d'absence de traumatisme du rein droit, l'animal est refermé plan par plan. Le plan costal est refermé au câble d'acier inoxydable par des points transfixants. Chaque plan musculaire est refermé par deux séries de points séparés, passés dans les aponévroses profondes et superficielles de chaque muscle sectionné. Le plan cutané est fermé au lin 2 par des points séparés largement passés. La moitié de ces points est enlevée au bout d'une semaine, l'autre douze à quinze jours plus tard.

$\mathrm{Il}$ est arrivé, dans les premières interventions, de rencontrer des accidents septiques postopératoires; abcès cutanés, nécrose ostéomyélitique... La surveillance rigoureuse de l'asepsie de chaque temps opératoire des dernières interventions nous permet, devant les résultats obtenus, de ne pas pratiquer une antibiothérapie systématique.

La surrénalectomie droite est, comme l'ont aussi dit KoLB et al. (1962), bien tolérée par la Truie qui est ingambe six heures après la fin de l'intervention.

Vingt jours au plus tôt après cette intervention l'animal subit la surrénalectomie gauche.

Annales de Biologie animale. - I972. 
Cette opération est généralement plus facile que la première, mais on peut rencontrer un accolement d'un pôle de la glande sur le flanc ventral de la veine rénale gauche ou de l'aorte.

Après surrénalectomie totale, une thérapeutique substitutive est immédiatement instaurée: après l'opération l'animal reçoit roo $\mathrm{mg}$ d'acétate de désoxycorticostérone (DOCA), puis tous les jours 50 à $75 \mathrm{mg}$ de DOCA par voie intramusculaire. Les animaux ont en plus quotidiennement $25 \mathrm{~g}$ de chlorure de sodium et $25 \mathrm{~g}$ de chlorure de potassium dans leur alimentation ou eau de boisson.

La survie des Truies, la poursuite de leur gestation et éventuellement leur parturition ont pu être ainsi obtenues. L'absence de traitement à base de corticostéroïdes entraîne la mort de l'animal.

\section{RÉSULTATS ET DISCUSSION}

L'excrétion urinaire des œstrogènes chez la Truie gravide présente un premier maximum entre le $25^{\mathrm{e}}$ et le $3^{\mathrm{I}}$ jour de gestation. Elle baisse ensuite jusque vers les $70^{\mathrm{e}}-75^{\mathrm{e}}$ jours, puis commence à remonter pour atteindre ses valeurs maximales au cours des derniers jours de gestation (RombauTs, I962). Cette cinétique d'élimination nous a conduits à pratiquer la surrénalectomie totale, soit $1 \mathrm{e} 22^{\mathrm{e}}$ jour de gestation $\left(\mathrm{J}_{22}\right)$, soit après le $9^{\mathrm{e}}$ jour. Le tableau $\mathrm{I}$ indique les différentes conditions expérimentales. La Truie 025 a été en plus ovariectomisée le $5^{\mathrm{e}}$ jour de gestation $\left(\mathrm{J}_{5}\right)$; sa gestation a été maintenue par l'injection intramusculaire de $300 \mathrm{mg}$ de progestérone en solution huileuse quotidiennement.

TABLEAU I

Conditions expérimentales

\begin{tabular}{|c|c|c|c|c|c|c|}
\hline \multirow{2}{*}{ Animaux } & \multirow{2}{*}{$\begin{array}{l}\text { Date de la } \\
\text { saillie }\end{array}$} & \multicolumn{2}{|c|}{ Surrénalectomie } & \multirow{2}{*}{ Ovariectomie } & \multirow{2}{*}{$\begin{array}{c}\text { Parturition* } \\
\text { ou } \\
\text { abattage** }\end{array}$} & \multirow{2}{*}{$\begin{array}{c}\text { Nombre de } \\
\text { porcelets ou } \\
\text { embryons }\end{array}$} \\
\hline & & droite & gauche & & & \\
\hline 9131 & $\begin{array}{c}10-2-70 \\
\left(\mathrm{~J}_{0}\right)\end{array}$ & $\begin{array}{c}11-2-70 \\
\left(J_{1}\right)\end{array}$ & $\begin{array}{l}4-3-70 \\
\left(J_{22}\right)\end{array}$ & - & $\begin{array}{c}13-3-70^{* *} \\
\left(J_{35}\right)\end{array}$ & 10 \\
\hline 025 & $\begin{array}{c}16-9-70 \\
\left(\mathrm{~J}_{0}\right)\end{array}$ & $3-9-70$ & $\begin{array}{l}8-10-70 \\
\left(J_{22}\right)\end{array}$ & $\begin{array}{c}21-9-70 \\
\left(J_{5}\right)\end{array}$ & $\begin{array}{c}26-10-70^{* *} \\
\left(J_{40}\right)\end{array}$ & 9 \\
\hline 872 & $\begin{array}{c}31-1-69 \\
\left(\mathrm{~J}_{0}\right)\end{array}$ & $\begin{array}{c}18-3-69 \\
\left(J_{46}\right)\end{array}$ & $\begin{array}{l}6-5-70 \\
\left(J_{95}\right)\end{array}$ & - & $\begin{array}{c}25-5-69 * \\
\left(\mathrm{~J}_{114}\right)\end{array}$ & 10 \\
\hline 813 & $\begin{array}{c}10-2-69 \\
\left(J_{0}\right)\end{array}$ & $\begin{array}{c}25-3-69 \\
\left(\mathrm{~J}_{43}\right)\end{array}$ & $\begin{array}{c}20-5-69 \\
\left(J_{98}\right)\end{array}$ & - & $\begin{array}{l}1-6-69 * \\
\left(J_{111}\right)\end{array}$ & 12 \\
\hline
\end{tabular}

Après surrénalectomie totale en début de gestation, l'excrétion d'œestrone est importante autour $\mathrm{du} 28 \mathrm{e}$ jour de gestation et comparable à celle des animaux gravides intacts. Une de ces truies ( $\mathrm{n}^{\circ} 9 \mathrm{I} 3 \mathrm{I}$ ) présente même un maximum de $7,44 \mathrm{mg}$ d'œstrone par 24 heures, valeur que nous n'avions jusqu'alors jamais constatée (fig. 2). En effet, les quantités habituellement excrétées à ce stade de gestation sont de 2 à $2,5 \mathrm{mg}$ par 24 heures; c'est ce que nous avons trouvé chez le deuxième animal $\left(\mathrm{n}^{\circ} 025\right): 2,75 \mathrm{mg} / 24$ heures (fig. 3 ). 


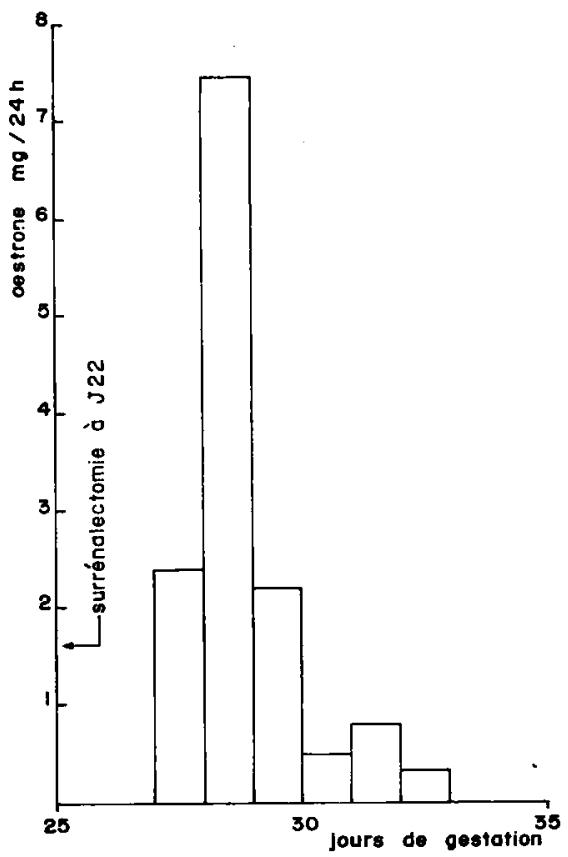

FIG. 2. - Estrone urinaire à la fin du premier mois de gestation : Truie 9ז3I

En fin de gestation, la surrénalectomie totale n'entraîne pas de chute de l'excrétion urinaire d'œstrone (fig. 4 et 5 ). La cinétique d'élimination est la même que celle qui est décrite chez les animaux gravides intacts (fig. 6). Les valeurs maximales

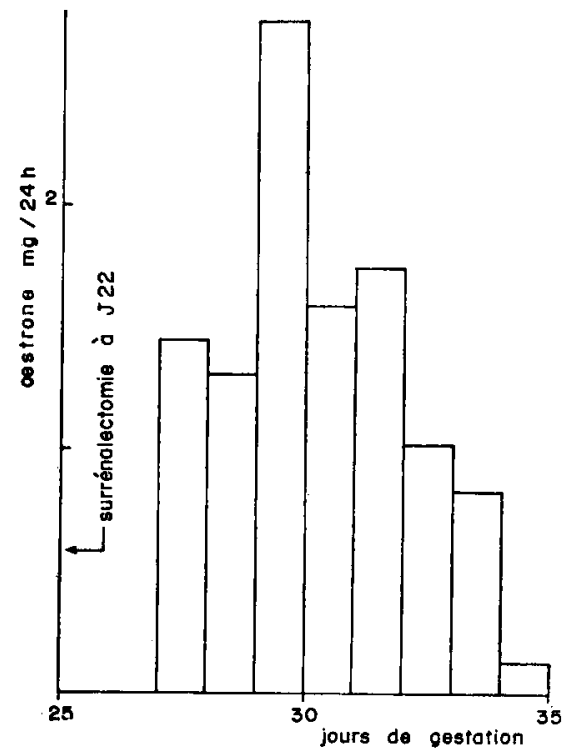

FIG. 3. - Estrone urinaire à la fin du premier mois de gestation : Truie 025 
de fin de gestation sont en particulier les mêmes qu'il y ait eu surrénalectomie totale ou non.

Dans l'espèce humaine, seules les observations de HAUSkNECHT (I965) sont identiques aux nôtres; en effet, après mise en silence des surrénales par la dexaméthasone, l'auteur n'observe de diminution ni dans le taux de production de l'œstradiol

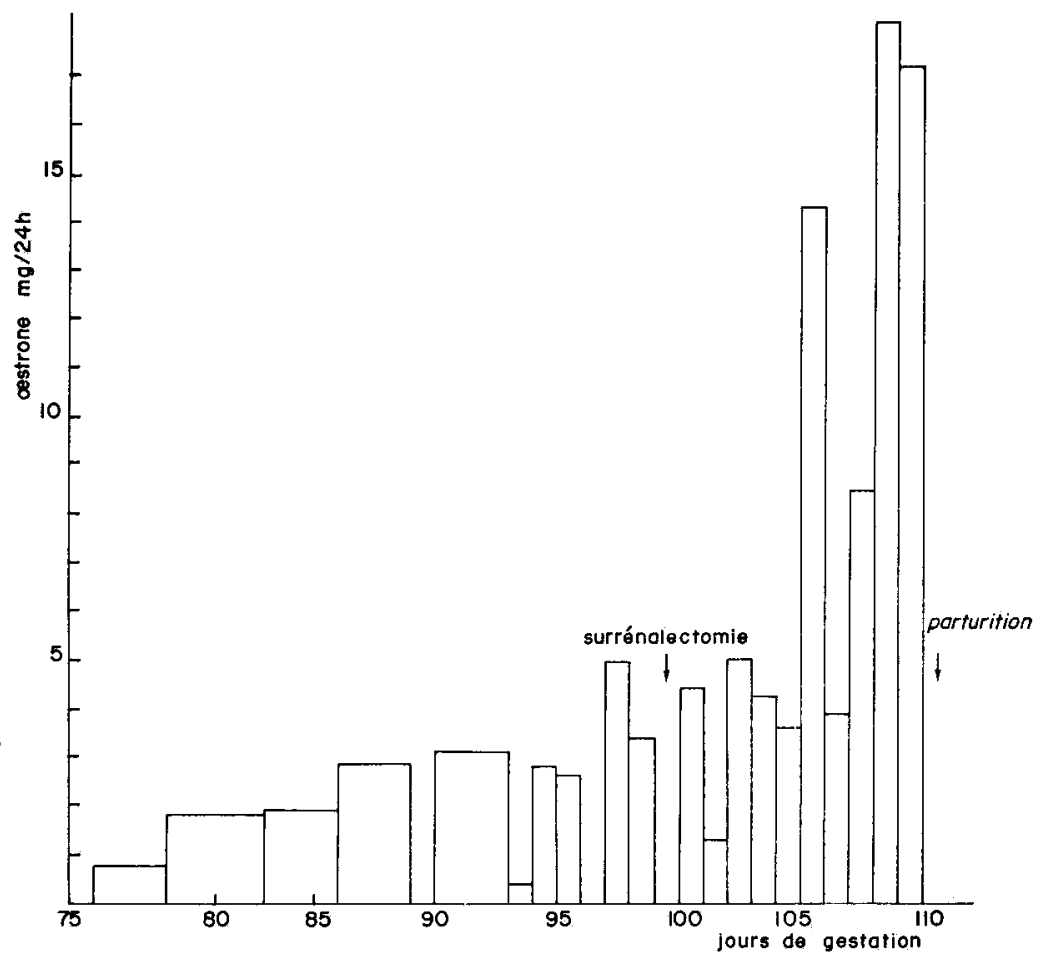

FIG. 4. - Estrone urinaire en fin de gestation après surrénalectomie : Truie 8I3

I7 $\beta$, ni dans l'excrétion urinaire d'œstriol. En revanche, MAC Donald et Sirteri (I965) ont trouvé, après traitement à la déxaméthasone, une nette diminution du taux de production d'œstradiol $\mathrm{I} 7 \beta(2,7 \mathrm{mg} / 24$ heures contre $7,5 \mathrm{mg} / 24$ heures$)$. Hammerstein et Nevinny-Stickei, (I965), sur une Femme surrénalectomisée, ont mis en évidence une forte baisse par rapport aux valeurs habituelles de l'excrétion urinaire des oestrogènes pendant le dernier mois de la grossesse : l'excrétion totale des trois œestrogènes classiques ne dépasse pas $5 \mathrm{mg} / \mathrm{jour}$, alors que 1'excrétion normale d'œestriol atteint à elle seule 25 à $30 \mathrm{mg}$ par jour chez les sujets normaux. WARREN et ChEATUM (I967) par suppression temporaire des surrénales, obtiennent au cours du troisième trimestre de gestation, une chute des œestrogènes urinaires de 50 à 90 p. IOO. HarknEss et al. (I966) chez une Femme surrénalectomisée ont également observé des valeurs basses de l'excrétion urinaire d'œstrogènes et ont estimé que les surrénales maternelles sécrètent environ la moitié des quantités de précurseurs nécessaires à la biosynthèse des œestrogènes pendant la grossesse. Ces résultats ont été confirmés par CharL,ES et al. (I970) sur la même Femme au cours de trois grossesses successives. 


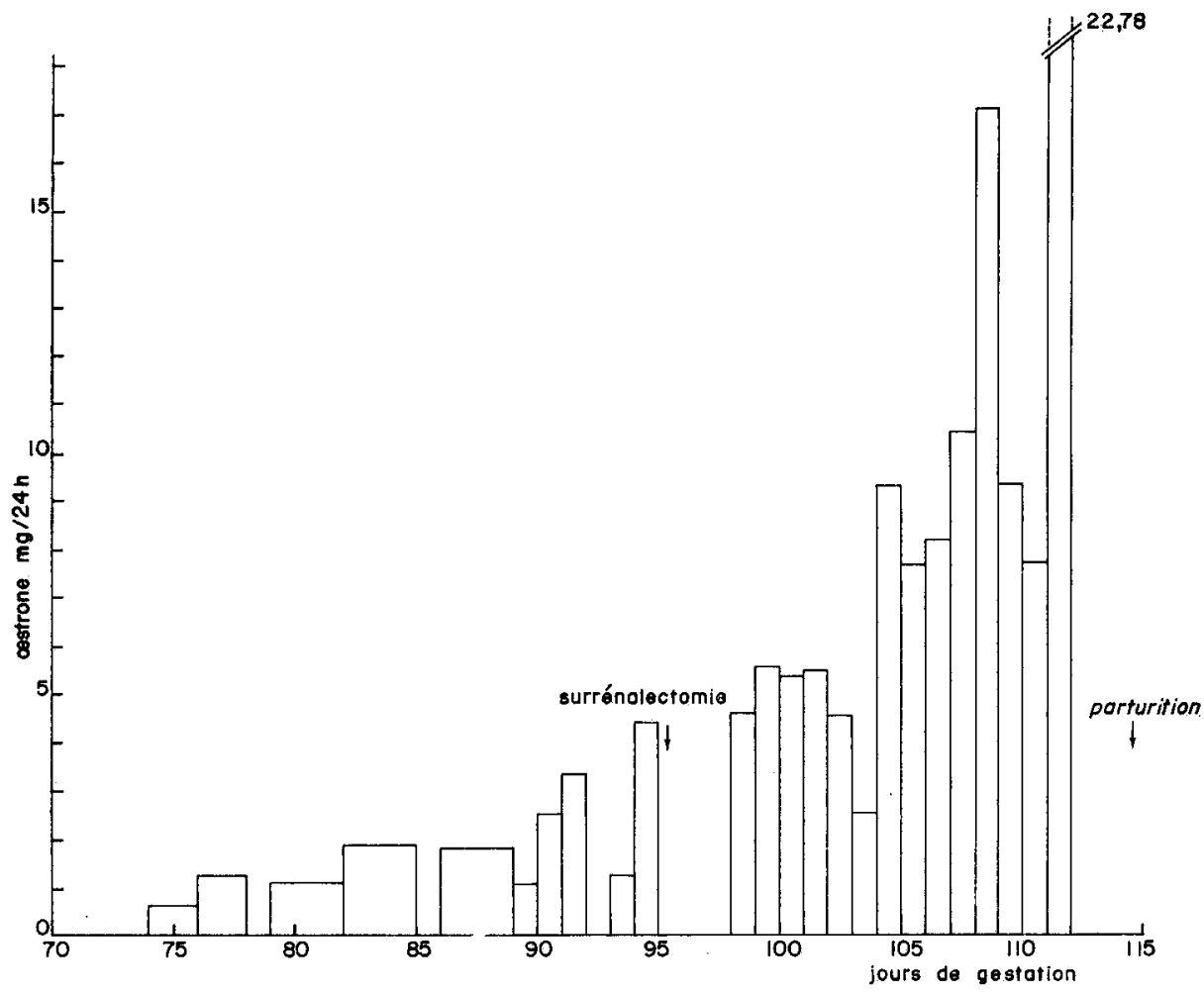

FIG. 5. - Estrone urinaive en fin de gestation après surrénalectomie : Truie 872

Récemment d'ailleurs, GURPIDE et VANDE WIELE (I97I) ont constaté que l'administration par voie orale de $60 \mathrm{mg}$ par jour de sulfate de déhydroépiandrostérone à une Femme surrénalectomisée enceinte, provoquait une augmentation du taux de production d'œstrone plus œstradiol de 5 à I6 mg par jour.

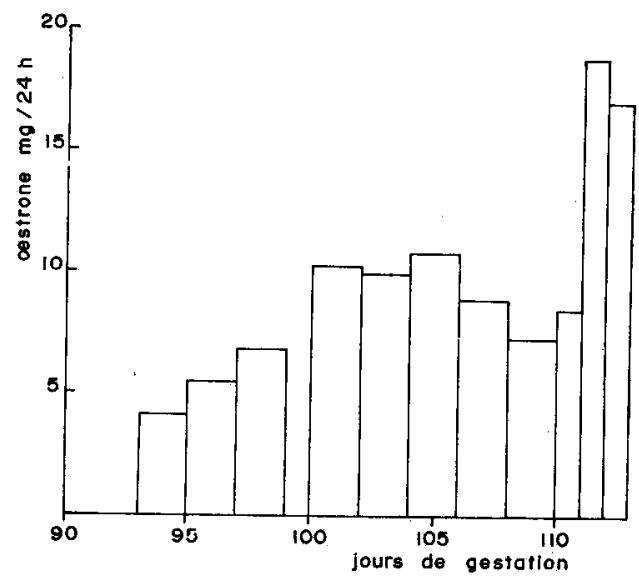

FrG. 6. - Ostrone urinaire en fin de gestation chez une Truie intacte 
Ces derniers résultats mettent bien en évidence le rôle important chez la Femme, des surrénales maternelles dans la biosynthèse des cestrogènes pendant la gestation.

Nos résultats montrent, au contraire, que chez la Truie cette synthèse peut s'effectuer pendant la gestation, en l'absence de précurseurs stéroïdiens surrénaliens maternels. Pour la production des cestrogènes, l'ensemble fœeto-placentaire doit probablement utiliser des molécules maternelles plus simples qui peuvent franchir plus aisément la barrière placentaire de type épithéliochorial dans le sens mère-foetus.

Reçu pour publication en avril 1972.

\title{
REMERCIEMENTS
}

Nous tenons à remercier les Éts Roussel-Uclaf qui mirent gracieusement à notre disposition les quantités de DOCA nécessaires à la bonne marche de nos expériences.

\section{SUMMARY}

\author{
ROLE OF MATERNAL ADRENAL, GLANDS
}

IN CESTROGEN PRODUCTION IN THE PREGNANT SOW

The quantities of œstrone excreted in sow urine increase considerably at the end of the first month of pregnancy and beginning at day 75. In order to know the importance of maternal adrenal steroids in fœeto-placental œstrogen synthesis, we excised the adrenals of four sows at different times during pregnancy (table $I$ ).

The surgical excision technique is described. It is always practised in two parts : excision of the right adrenal first, followed by that of the left adrenal at least 20 days later. The survival of the animals is ensured by daily administration of desoxycorticosterone acetate, sodium chloride and potassium chloride. The animals die without substitutive treatment.

After total excision of the adrenals, urinary œestrone excretion presents a maximum at about day 28 of gestation as in intact pregnant animals (fig. 2 and 3). At the end of pregnancy, we find maximal elimination values of urinary cestrone, these values being the same whether the adrenals are excised or not (fig. 4,5 and 6).

In Woman, studies show that the maternal adrenals secrete at least one half of the precursors necessary to œstrogen biosynthesis during pregnancy. On the contrary, our results show that in the sow this fœto-placental synthesis may occur normally when maternal steroid precursors are absent.

\section{RÉFÉRENCES BIBLIOGRAPHIQUES}

Brown J. B., I955. A chemical method for the determination of cestriol, cestrone and cestradiol in human urine. Biochem. J, 60, I85-193.

Charles D., Harkness R. A., Kenny F. M., Menini E., Ismail, A. A. A., Durkin J. W., LoRAINE J.A., I970. Steroid excretion patterns in an adrenalectomized woman during three successive pregnancies. Am.J. Obstet. Gynec., 106, 66-74.

Fèvre J., 1970. Conversion en cestrone de quelques stéroïdes C-rg chez la Truie gestante. Ann. Biol. anim. Bioch. Biophys., 10, 25-35.

Fevre J., Leglise P.-C., Rombauts P., I968. Du rôle de l'hypophyse et des ovaires dans la biosynthèse des cestrogènes au cours de la gestation de la Truie. Ann. Biol. anim. Bioch. Biophys., 8, $225-233$.

GuRPIDE E., VANDE WIELE R. L., r97I. Utilization of ingested dehydroisoandrosterone sulfate for the production of estrogens by normal and adrenalectomized pregnant subjects. J. Ster. Biochem., 2, II-I8. 
Hammerstein J., Nevinny-Stickel R. L., I965. Verminderte Oestrogen-Ausscheidung in der Schwangerschaft nach subtotaler Adrenalectomie. Acta Endocr., 48, 375-382.

Harkness R. A., Menini E., Charles D., Kenny F. M., Rombaut R., ig66. Studies of urinary steroid excretion by an adrenalectomized woman during and after pregnancy. Acta Endocr., 52, 409-4I5.

Hausknecht R. U., 1965. Maternal dehydroepiandrosterone and estrogen production in late pregnancy, Obstet. Gynec., 26, 544-548.

Kolb E., Müller I., Seidel H., Ellrich T., I962. Untersuchungen an Schweinen. 2. Mitteilung. Untersuchungen über die Ausfallsercheinungen und über das Verhalten der Thorntester nach halbseitiger und totaler Adrenalektomie, unter besonderer Berücksichtigung der Natrium-und Kaliumkonzentration im Serum sowie in verschiedenen Abschnitten der Herz- und Skelettmuskulatur, $Z b l$. Vet. med., 9, 664-692.

Mac Donald P. C., Silteri P. K., r965. Origin of estrogen in women pregnant with an anencephalic fetus. J. Clin. Invest., 44, 465-474.

Rombauts P., 1962. Excrétion urinaire des œstrogènes chez la Truie pendant la gestation. Ann. Biol. anim. Bioch. Biophys., 2, 15I-156.

WarRen J. C., Cheatum S. G., r967. Maternal urinary estrogen excretion : effect of adrenal suppression. J. Clin. Endocr. Metab., 27, 433-436. 